\title{
Las Arterias Circunflejas Femorales en el Triángulo Femoral
}

\author{
The Circumflex Femoral Arteries in the Femoral Triangle
}

*Mariano del Sol; **Iván Suazo Galdames \& ****élgica Vásquez

DEL SOL, M.; SUAZO, G. I. \& VÁSQUEZ, B. Las arterias circunflejas femorales en el triángulo femoral. Int. J. Morphol., 29(2):644649, 2011.

RESUMEN: Conocer el origen y distribución de las arterias circunflejas femorales (AaCF) en el hombre, es importante en el momento quirúrgico de la reconstrucción vascular. Se disecó el contenido del triángulo femoral en 92 miembros inferiores de cadáveres formolizados, adultos, de sexo masculino y diferentes grupos étnicos, descubriéndose la arteria femoral (AF) y sus ramas originadas a nivel del triángulo femoral. Se localizó el origen de cada una de las AaCF determinándose el tipo y lugar de origen. La arteria circunfleja femoral medial (ACFM) se originó en 43 casos (46,7\%) desde la AF; en 41 casos $(44,6 \%)$ desde la arteria femoral profunda (AFP); en 7 casos $(7,6 \%)$ en un tronco común formado por la AFP y AaCF y en un caso $(1,1 \%)$ desde la arteria circunfleja femoral lateral (ACFL). La ACFM tenía en 75 casos $(81,5 \%)$ un origen más proximal que la ACFL y en 9 casos $(9,8 \%)$ su origen era al mismo nivel. La ACFL se originó en 68 casos $(73,9 \%)$ desde la AFP; en 17 casos $(18,5 \%)$ desde la AF; en 7 casos $(7,6 \%)$ en un tronco común formado por la AFP y AaCF. El origen de la ACFL fue considerado independientemente si su ramo descendente se originaba desde ella o lo hacía desde la AF. Debido a la presencia de una serie de elementos nobles, conocer el origen preciso de las arterias y sus eventuales variaciones, adquiere especial importancia en los procedimientos realizados en la región.

PALABRAS CLAVE: Anatomía; Arteria femoral; Arterias circunflejas femorales.

\section{INTRODUCCIÓN}

La arteria femoral (AF) y sus ramas tienen un importante rol en el aporte arterial del miembro inferior. Si esta arteria se obstruye, se puede restablecer la circulación del miembro inferior a través de las anastomosis que se realizan entre las arterias del triángulo femoral, región donde se originan la arteria circunfleja femoral medial (ACFM) y la arteria circunfleja femoral lateral (ACFL), ya sea, desde la AF, arteria femoral profunda (AFP), o en un tronco fémorocircunflejo, entre otras.

Además, en el triángulo femoral se originan las arterias pudendas externas, circunfleja iliaca superficial y, eventualmente, las arterias circunfleja iliaca profunda, epigástrica superficial y epigástrica inferior (del Sol et al., 1997). Junto a estas arterias, en el triángulo femoral se encuentran nervios, siendo el más importante el femoral, como también venas profundas que acompañan a las respectivas arterias y venas superficiales que, perforando la fascia cribiforme, terminan en la vena femoral.
Para numerosos autores, el origen de las arterias circunflejas femorales (AaCF) es muy variable. Según Gremigni (1968) y Siddharth et al. (1985) estas arterias pueden nacer aisladamente o por un tronco común, ya sea de la AF o de la AFP, pudiendo ser dobles o bien faltar, y son reemplazadas como lo indicó Orts LLorca (1952) por arterias vecinas.

Autores como Adachi (1928) han señalado orígenes muy diversos para las $\mathrm{AaCF}$, ya sea en la arteria iliaca externa o en un tronco común con las arterias obturatriz y epigástrica inferior.

De manera general, la ACFM se origina de la AFP, da cinco ramas: superficial, profunda, acetabular, ascendente y descendente. La arteria en su trayecto, irriga a los músculos aductores, rodea medialmente al fémur, pasando primero entre los músculos pectíneo y psoas mayor y luego entre los músculos obturador externo y aductor cor-

\footnotetext{
* Facultad de Medicina, Universidad de La Frontera, Temuco, Chile.

** Facultad de Ciencias de la Salud, Universidad de Talca, Chile.

${ }^{* * * *}$ Facultad de Ciencias de La Salud, Universidad Autónoma de Chile, Chile.
} 
to para, finalmente, pasar entre el músculo cuadrado femoral y el margen superior del músculo aductor magno. La ACFL da tres ramas, ascendente, descendente y transversa.

En una población turca Tanyeli et al. (2006) determinaron que en el 79\% de los casos la ACFM era una rama de la AFP y en el $15 \%$ su origen era en la AF. En el $4 \%$ la ACFM era doble. En cada uno de estos casos una arteria provenía de la AF y la otra de la AFP. En uno de estos casos también la ACFL era doble. Además, encontraron en un caso un tronco común entre la ACFM y AFP y en otro caso un tronco común entre las dos AaCF y la AFP.

Según Siddharth et al. en menos de la mitad de los casos, la ACFM se origina independientemente de la AF. Su origen en la AFP es de $73 \%$ según Orts LLorca y de 63\% según Testut \& Lartarjet (1954). En cambio Leborgne (1974) y Marcade et al. (1978) informaron un 50,5 y 54\%, respectivamente.

Para Warwick \& Williams (1975) cuando la ACFM se origina de la AFP, lo hace desde su cara posteromedial, sin embargo, estos autores señalan que puede originarse de la AF.

Según Orts LLorca cuando la ACFM se origina de la AF lo hace a una distancia aproximada a $\operatorname{los} 0,5 \mathrm{~cm}$, en cambio cuando se orgina de la AFP esta distancia se eleva a $1,0 \mathrm{~cm}$ del origen de la arteria.

Un estudio de Prakash et al. (2010) reportó que la ACFM en el $67,2 \%$ se originaba a partir de la AFP, mientras que en el 32,8\% de los casos se originaba desde la AF. Por otro lado, la ACFL en el $81,25 \%$ se originaba de AFP, mientras que en el 18,75\% lo hacía de la AF.

De acuerdo a los estudios de Siddhart et al., cuando no existe la ACFL, ésta es reemplazada por una segunda ACFM. Incluso se ha descrito que una rama con origen en la arteria iliaca externa entraba en el triángulo femoral, pasaba posteriormente a la vaina femoral y daba irrigación a los músculos, anastomosándose con la ACFM (Nayak, 2008).

Fukuda et al. (2005)) evaluaron las variantes anatómicas de la ACFL en arteriografías femorales obtenidas antes de la cirugía coronaria en 131 pacientes adultos. El patrón más común fue el origen de la ACFL desde la AFP $(78,6 \%)$ y el injerto arterial fue seleccionado de ese patrón en el 92,3\% de los pacientes en los que se utilizó la rama descendente de la ACFL para el bypass de arteria coronaria.
Desde el punto de vista anatomo-quirúrgico el conocimiento de estos vasos adquiere importancia en múltiples procedimientos entre los cuales se puede mencionar a la rama descendente de la arteria circunfleja femoral lateral la cual se ha utilizado como un nuevo injerto arterial de bypass de arteria coronaria.

Por lo anterior, considerando la presencia de una serie de elementos nobles a nivel del triángulo femoral, se realizó este estudio para determinar el origen preciso de las AaCF y sus eventuales variaciones, considerando además, la especial importancia de este conocimiento en los diversos procedimientos que se realizan en la región, especialmente, en las cirugías de reconstrucción vascular.

\section{MATERIAL Y MÉTODO}

En 92 miembros inferiores de cadáveres formolizados, adultos, de sexo masculino y diferentes grupos étnicos, se disecó el triángulo femoral, descubriéndose la $\mathrm{AF}$ y las ramas originadas de esta arteria. Se localizó el origen de cada una de las $\mathrm{AaCF}$ determinándose el tipo de origen, ya sea, de un tronco común o separadas y el lugar espacial de su origen. Además, se observó el nivel de origen de las $\mathrm{AaCF}$ y se comparó éste, entre ambas arterias.

\section{RESULTADOS}

La ACFM se originó en 43 casos $(46,7 \%)$ directamente de la AF; en 41 casos $(44,6 \%)$ desde la AFP; en 7 casos $(7,6 \%)$ en un tronco común formado por las AFP y $\mathrm{AaCF}$ y en un caso $(1,1 \%)$ desde la ACFL. En este caso la ACFM se originaba de la parte medial de la ACFL, se dirigía hacia distal y luego girando hacia medial, cruzaba posteriormente a la AFP. En la Figs. 1 y 2 y Tabla I se observa el origen de la ACFM.

La ACFL se originó en 68 casos $(73,9 \%)$ directamente de la AFP; en 17 casos $(18,5 \%)$ desde la AF; en 7 casos $(7,6 \%)$ en un tronco común formado por las AFP y AaCF. En las Figs. 1 y 2 y Tabla I se observa el origen de la ACFL.

En 9 casos $(9,8 \%)$ el origen de ambas AaCF era al mismo nivel. La ACFM tenía en 75 casos $(81,5)$ un origen más proximal que la ACFL.

El origen de la ACFL fue considerado independientemente si su ramo descendente se originaba desde ella o lo hacía desde la arteria femoral. 

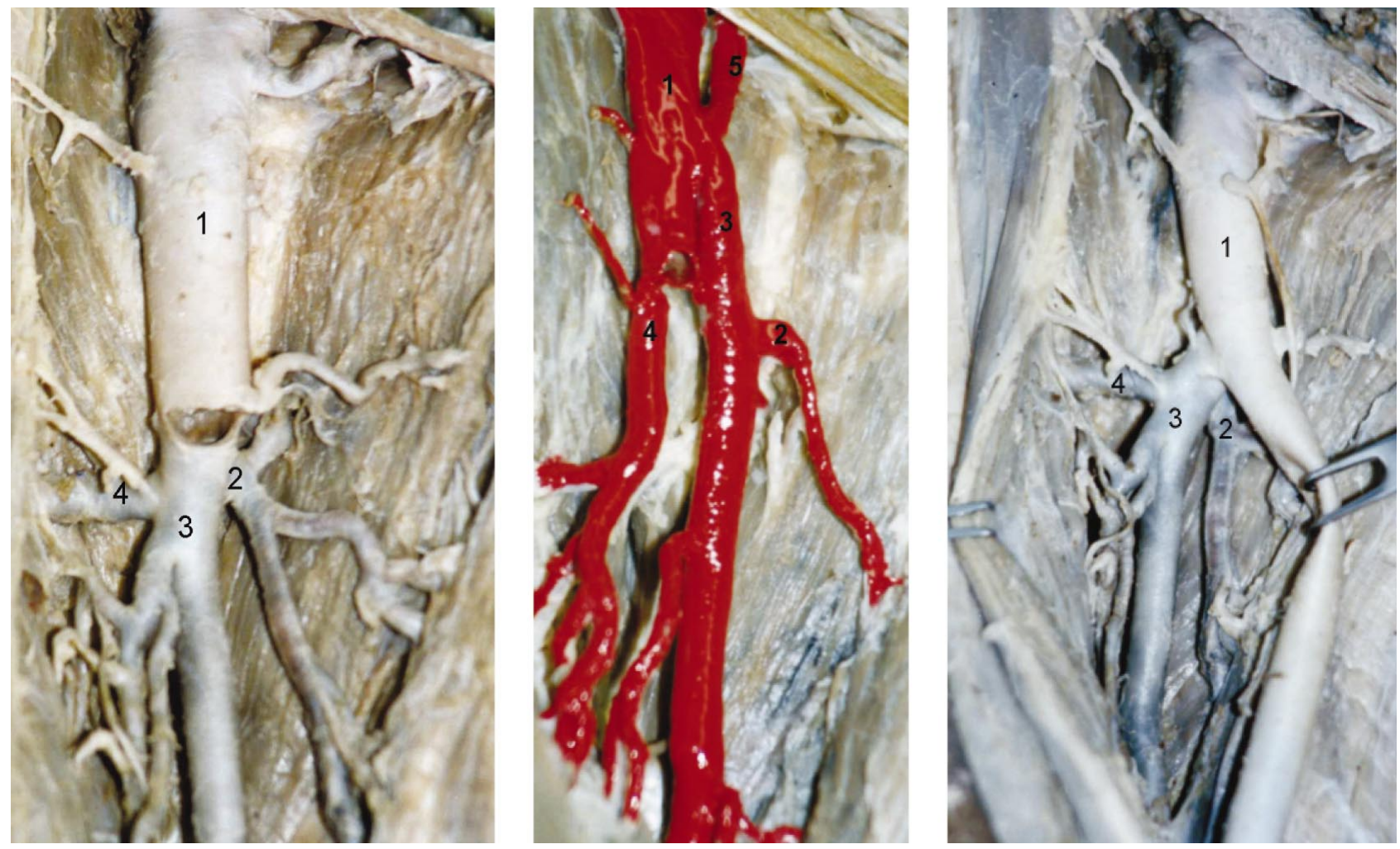

Figs. 1a,b,c. Origen de las arterias circunflejas femorales medial y lateral, en 92 miembros inferiores de individuos adultos, de ambos sexos y diferentes grupos étnicos. 1. Arteria femoral; 2 Arteria circunfleja femoral medial; 3. Arteria Femoral profunda; 4. Arteria circunfleja femoral lateral; 5. Arteria epigástrica inferior (Variación de origen en la arteria femoral profunda).

Tabla I. Origen de las arterias circunflejas femorales medial y lateral, en 92 miembros inferiores de individuos adultos, de ambos sexos y diferentes grupos étnicos.

\begin{tabular}{lcccc}
\hline Origen & Arteria circunfleja femoral medial & \multicolumn{2}{c}{ Arteria circunfleja femoral lateral } \\
\hline & Número & $\%$ & Número & $\%$ \\
\hline Medial de la arteria femoral prof unda & 29 & 31,52 & - & - \\
Medial de la arteria femoral & 26 & 28,26 & - & - \\
Posteromedial de la arteria femoral & 10 & 10,87 & - & - \\
Posteromedial de la arteria femoral profunda & 9 & 9,78 & - & - \\
Posterior de la arteria femoral & 7 & 7,61 & & \\
Posterior de la arteria femoral profunda & 3 & 3,26 & & 7,61 \\
Tronco fémorocircunflejo & 7 & 7,61 & 7 & - \\
Arteria circunfleja femoral lateral & 1 & 1,09 & - & 40,22 \\
Posterolateral de la arteria femoral profunda & - & - & 37 & 33,69 \\
Lateral de la arteria femoral prof unda & - & - & 31 & 11,96 \\
Lateral de la arteria femoral & - & - & 11 & 6,52 \\
Posterolateral de la arteria femoral & - & - & 6 & \\
\hline
\end{tabular}

\section{DISCUSIÓN}

El colgajo de perforantes basado en la ACFL es uno de los más usados en la reconstrucción de partes blandas de pequeño y mediano tamaños. El aporte sanguíneo del colgajo depende de la rama descendente de la ACFL y es usa- 

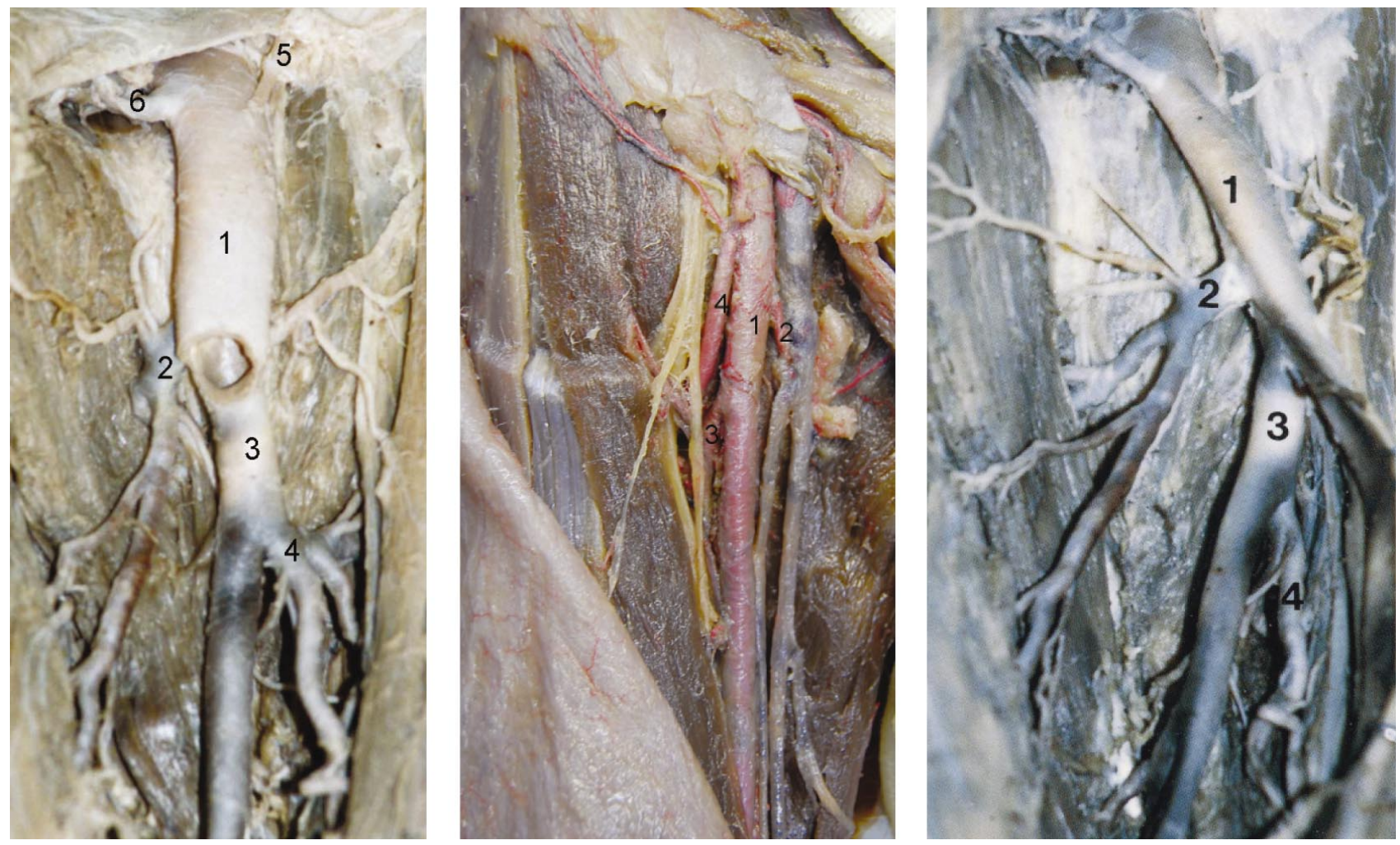

Figs. 2a,b,c. Origen de las arterias circunflejas femorales medial y lateral, en 92 miembros inferiores de individuos adultos, de ambos sexos y diferentes grupos étnicos. 1. Arteria femoral; 2 Arteria circunfleja femoral medial; 3. Arteria Femoral profunda; 4. Arteria circunfleja femoral lateral; 5. Arteria circunfleja iliaca profunda; 6. Arteria obturatriz.

do en reconstrucciones de cabeza y cuello (piso de la cavidad oral, labios, esófago, atrofia hemifacial, etc.) y en otros sitios como reconstrucción mamaria o donante del mismo miembro inferior.

Además, variaciones anatómicas de la ACFL han sido mencionadas como una hipótesis etiopatogénica de la lipoatrofia semicircular del muslo.

Por otra parte, la ACFM es considerada la fuente principal de la irrigaciónd de la cabeza femoral, así cuando existe osteonecrosis, se usan ramas ascendentes de la ACFL para su anastomosis con los vasos del tejido donante.

Se ha planteado por Sohier et al. (1964) que un origen común de las AaCF y FP, representaría una forma más progresiva, en cuanto la independencia de estas arterias sugeriría una forma más primitiva. En nuestro estudio, la no disociación de las AaCF y FP, se presentó en el 7,61\% de los casos, muy semejante al 5\% observado por Testut \& Latarjet y por del Sol et al. (1996) en el 6,25\% de las muestras examinadas. Por otra parte, la existencia de este tronco fémorocircunflejo profundo o perfecto se presentó, según Adachi, en el 63,2\% de los japoneses, cifra muy elevada en comparación con todos los estudios reportados.

Para algunos autores, la normalidad de origen de las AaCF es junto a la AFP, en un tronco común cuyo origen sería la AF. Esta disposición clásica alcanzaría a $38 \%$ según Leborgne y 39\% según Marcade et al.

Adachi en individuos japoneses reportó la existencia de un tronco fémorocircunflejo medial en el $14,4 \%$ de los casos.

No observamos un origen común de las AaCF desde la AF, como fue descrito en el 5\% de los casos por Testut \& Latarjet, cifra idéntica reportada por Siddharth et al. Sin embargo, en un caso pudimos observar a una ACFM originándose desde la ACFL, comparable o asimilable con la coexistencia de dos arterias circunflejas femorales mediales, una de las cuales reemplazaría a la ACFL ausente.

Para Siddharth et al. el origen de las AaCF es en la AFP (67\% ACFL y 63\% ACFM). En menor porcentaje el origen de estas arterias está en la AF (16\% ACFL y $26 \%$ ACFM). Sin embargo, en el 5\% de los casos ambas AaCF se originaban en un tronco común desde la AF. 
Lipshutz propuso en 1916 clasificaciones para el origen de las AaCF. Para este autor, en el Tipo I (40\%) las AaCF se originan, separadamente, de la AFP; en el Tipo II (25\%) solo la ACFM se origina de la AF; en el Tipo III (19\%), solamente la ACFM se origina de la AFP y en el Tipo IV (11\%) ambas AaCF lo hacen desde la AF. De esta manera, en el 59\% de los casos (Tipos I y III) la ACFM tiene su origen en la AFP y en el 65\% la ACFL se origina en la AFP.

Williams et al. (1939) propusieron diversos tipos de origen de las $\mathrm{AaCF}$ a nivel del triángulo femoral. En los tipos 1, 1a y 2, en los cuales la ACFM se origina directamente de la AF, se observó en el 46,7\% de los casos, inferior al porcentaje encontrado por estos mismos autores $(25,5 \%)$ en individuos blancos y negros, a los datos reportados por Adachi en japoneses $(20,1 \%)$ y en chinos por P'an Ming-Tzu (1937) en promedio de $20 \%$.

Por otra parte, ocurre algo similar si agrupamos los Tipos 3, 4a y 5, donde el origen de la ACFM se realiza directa o indirectamente desde la AFP, o en un tronco común con la ACFL. Estos tipos de orígenes de la ACFM se presentó en el 52,1\% de nuestra muestra, si lo comparamos con el 75\% de Williams et al., en individuos blancos y negros, el 78,7\% reportado por Adachi en japoneses y al 79,9\% obtenido por P' an Ming-Tzu en los chinos y en el 53,1\% por del Sol et al. (1996).

Uzel et al. (2008) investigaron el origen de la ACFL en la población turca. Ellos determinaron que en el 77,3\% de los casos se originaba de la AFP y en el 19,1\% de los casos lo hacía desde la AF. Señalaron también el origen distinto de las ramas ascendente y descendente de la ACFL, originándose en el 1,8\% separadamente desde las AFP y AF. En un $0,9 \%$ la ACFL se originaba en un tronco común con la AFP y en otro $0,9 \%$ se originaba en un tronco común entre ambas AaCF y la AFP.
Para Siddharth et al., en más de la mitad de los casos, la AFP no da origen a la ACFM, naciendo ésta independientemente desde la $\mathrm{AF}$, descripción que concuerda con nuestros resultados ya que sólo en el 44,56\% el origen de esta arteria se realizó directamente de la AFP y en el 46,74\% desde la AF.

No observamos formaciones como las descritas por Nasu \& Chiba (2009) donde las ACFL y arteria circunfleja iliaca profunda formaban un tronco común o un tronco formado por las ACFM y aa. epigástrica inferior y obturatriz.

En un caso descrito por Ciftcioglu et al. (2009) la ACFM se originaba de la parte posterolateral de la AF, $32 \mathrm{~mm}$ distal al ligamento inguinal. Sin embargo, la emergencia señalada por estos autores y su curso posterior, no es frecuente, de acuerdo a lo observado en nuestro estudio.

En conclusión, el patrón de origen de las AaCF es muy diverso, como es posible observar en los reportes de diferentes estudios realizados en distintos grupos étnicos. Sin embargo, es posible asegurar que la ACFL, normalmente, tiene su origen de manera independiente en la AFP $(73,91 \%)$, concordando con la mayoría de los datos encontrados en la literatura. Por otra parte, el origen de la ACFM, puede darse tanto desde la AF $(46,7 \%)$ o desde la AFP $(44,6 \%)$, constituyendo prácticamente sólo dos patrones de origen. Un patrón menos común es el origen en un tronco femorocircunflejo, formado por la AFP y las AaCF.

Por lo anterior, el conocimiento anatómico de las variaciones de la $\mathrm{AF}$ a nivel del triángulo femoral, como también de la AFP, ACFM y ACFL resulta fundamental para minimizar las eventuales complicaciones quirúrgicas que se pueden producir en la región. Asimismo, la identificación de variaciones anatómicas de estas $\mathrm{AaCF}$ es importante para los radiólogos y cirujanos, debiéndose dar suficiente énfasis a la enseñanza de la anatomía de la región.

DEL SOL, M.; SUAZO, G. I. \& VÁSQUEZ, B. The circumflex femoral arteries in the femoral triangle. Int. J. Morphol., 29(2):644$649,2011$.

SUMMARY: It is important to identify the origin and distribution of the circumflex femoral arteries (CFA) at the time of vascular reconstructive surgery. The femoral triangle contents in 92 lower extremities of formolized adult male cadavers of different ethnic groups, were dissected uncovering the femoral artery (FA) and its branches originating at the level of the femoral triangle. The origin of each CFA was identified determining the origin type and location. The medial circumflex femoral artery (MCFA) originated from the FA in 43 cases (46.7\%); from the profunda femoris artery (PFA) in 41 cases (44.6\%); from a common trunk formed by the PFA and CFA in 7 cases $(7.6 \%)$, and in one case (1.1\%) from the lateral circumflex femoral artery (LCFA). In 75 cases (81.5\%) the MCFA was most proximal than the LCFA, and in 9 cases $(9.8 \%)$ it originated at the same level. The LCFA originated at the PFA in 68 cases (73.9\%); from the FA in 17 cases (18.5\%); from a common trunk formed by the PFA and CFA in 7 cases $(7.6 \%)$. The origin of the LCFA was considered regardless, whether the descending branch originated therein or from the FA. Considering the presence of a number of important elements it is essential to identify the precise origin of the arteries and its eventual variations in procedures carried out in that area.

KEY WORDS: Anatomy; Femoral artery; Circumflex femoral arteries. 


\section{REFERENCIAS BIBLIOGRÁFICAS}

Adachi, B. Das Arteriensystem der Japaner. Kyoto, 1928.

Del Sol,; Olave, E. \& Mandiola, E. Origen anómalo de la arteria epigástrica inferior. Rev. Chil. Cs. Méd. Biol., $7(1): 13-16,1997$.

Del Sol, M.; Olave, E.; Vieira, M. C. \& Collipal, E. Origen de la arteria circunfleja femoral medial. Rev. Chil. Cs. Méd. Biol., 6(1):49-55, 1996.

Ciftcioglu, E.; Kale, A.; Kopuz, C.; Edizer, M.; Aydin, E. \& Demir, M.T. Medial circumflex femoral artery with different origin and course: a case report and review of the literature. Folia Morphol (Warsz), 68(3):188-91, 2009.

Fukuda, H.; Ashida, M.; Ishii, R.; Abe, S. \& Ibukuro, K. Anatomical variants of the lateral femoral circumflex artery: an angiographic study. Surg. Radiol. Anat., 27(3):260-4, 2005.

Gremigni, D. Sulla origine di alcuni rami collaterali dellárteria femorale. Zeitschrift für Anatomie un Entwicklungsgeschichte, 127:42-54, 1968.

Kasai, T.; Suzuki, T.; Fukushi, T.; Kodama, M. \& Chiba, S. Peripheral distribution of the medial circumflex femoral artery. Okajimas Folia Anat., Jp., 60:89-97, 1985.

Leborgne, J. Considerations sur les rapports de l'artère femorale profonde et de ses branches. Bull. Asoc. Anat., 58:137-48, 1974.

Lipshutz, B. B. Studies on the blood vascular tree. A composite study of the femoral artery. Anat. Rec., 10(5):361-70, 1916.

Marcade, E.; Leguerrier, A.; Scarabin, J. M.; Rioux, C.; Logeais, Y. \& Lanchou, G. L'artère femorale profonde. Étude anatomoradiologique. Bull. Assoc. Anat., 62:1439, 1978.

Nasu, H. \& Chiba, S. Rare case of femoral artery ramification and origin of the obturator artery. Anat. Sci. Int., 84(4):323-6, 2009.

Nayak, B. Satheesha. Abnormal branch of external iliac artery in the iliac fossa. Int. J. Morphol., 26(2):445-6, 2008.
Orts LLorca, F. Anatomía humana. Barcelona, Científico Médica, 1952.

P'an Ming-Tzu. Origin of deep and circumflex femoral group of arteries in the Chinese. Am. J. Physical Anthropol., 22(3):417-24, 1937.

Prakash; Kumari, J.; Kumar Bhardwaj, A.; Jose, B. A; Kumar Yadav, S. \& Singh, G. Variations in the origins of the profunda femoris, medial and lateral femoral circumflex arteries: a cadaver study in the Indian population. Rom. J. Morphol. Embryol., 51(1):167-70, 2010.

Siddhart, P.; Smith, N. L.; Mason, R. A. \& Girón, F. Variational anatomy of deep femoral artery. Anat. Rec. 212: 206-9, 1985.

Sohier, H. M. L.; Lafont, S.; Fustec, R. \& Le Guyader, A. Étude anthropologique de l'artère fémorale en Afrique Occidentale. Bull. Asoc. Anat., 120:1236-43, 1964.

Tanyeli, E.; Uzel, M.; Yildirim, M. \& Celik, H. H. An anatomical study of the origins of the medial circumflex femoral artery in the Turkish population. Folia Morphol. (Warsz)., 65(3):209-12, 2006.

Testut, L. \& Latarjet, A. Tratado de anatomía humana. 9 ed. Barcelona, Salvat, 1954.

Uzel, M.; Tanyeli, E. \& Yildirim, M. An anatomical study of the origins of the lateral circumflex femoral artery in the Turkish population. Folia Morphol (Warsz), 67(4):226-30, 2008.

Warwick, R. \& Williams, P. Gray Anatomia. 35. ed. Rio de Janeiro, Guanabara \& Koogann 1975.

Williams, G. D.; Sindelar, R. J.; Peart, J. C.; Martin, L. R. McIntire, L. R. \& Martin, C. H. Origin of the deep and circumflex femoral group of arteries. Anat. Rec., 46:2769, 1939.

Dirección para correspondencia:

Prof. Dr. Mariano del Sol

Facultad de Medicina

Universidad de La Frontera

Casilla 54-D

Temuco - CHILE

Email: mdelsol@ufro.cl

Recibido : 24-02-2011

Aceptado: 12-04-2011 